\title{
CARGA MENTAL DE TRABAJO EN ENFERMERAS/OS DE UNIDADES DE PACIENTE CRÍTICO: ESTUDIO PILOTO
}

\author{
MENTAL WORKLOAD IN NURSES CRITICAL PATIENT UNIT: PILOT STUDY
}

PAULA CEBALLOS VÁSQUEZ*

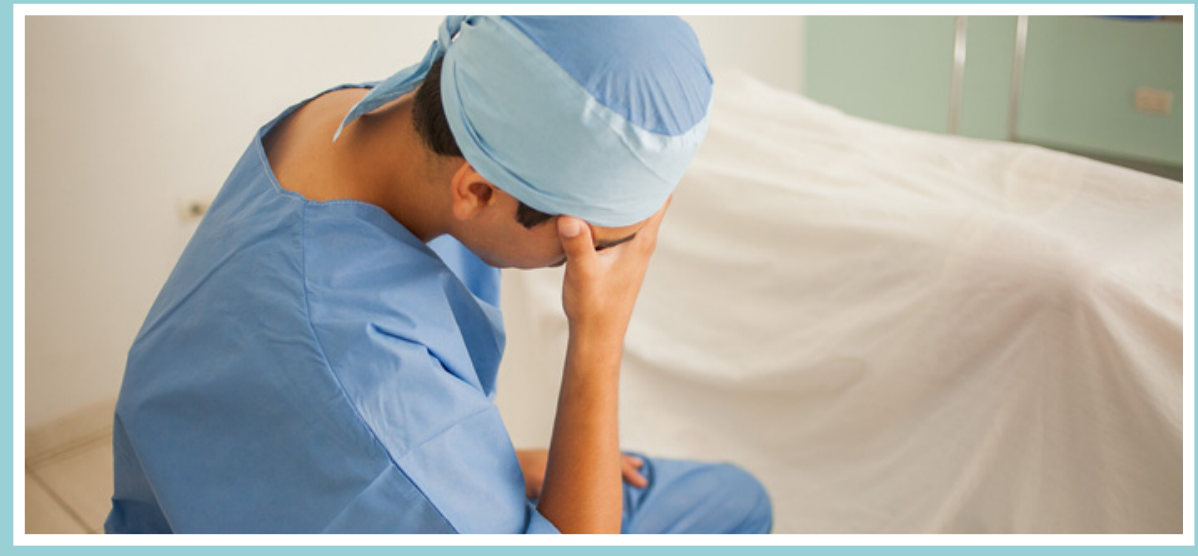

\section{ABSTRACT}

Objective: To determine the perception of mental workload on nurses as a critical care unit of a Regional Hospital in Chile. Methods: descriptive study, transverse and exploratory design. 10 nurses were censused who work in a turn of the Critical Patient Unit of the Regional Hospital of Chile. Scale of Subjective Mental Workload and bio/psycho/ socio/demographic background: To collect data two instruments were used. To carry out this study, the Emanuel E. ethical requirements were considered. Results: average 3.8 (SD = 66) workers / UPC as a participant in this study, perceived mental workload when performing their work. Conclusion: Mental work overload, could generate consequences for their physical and mental health of nurses / participants, making it a priority to take measures to maintain adequate mental burden.

Keywords: Mental load, Occupational Health Nursing

\section{RESUMEN}

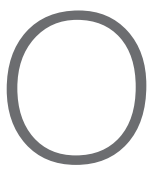
bjetivo: determinar la percepción de carga mental de trabajo en los enfermeros/as de una unidad de pacientes críticos de un Hospital Regional en Chile. Material y método: estudio con diseño descriptivo, trasversal y exploratorio. Se censó a 10 enfermeras/os que se desempeñan en un turno de la Unidad de Pacientes Críticos de un Hospital Regional de Chile. Para recopilar los datos se utilizaron dos instrumentos: Escala Subjetiva de Carga Mental de Trabajo y Antecedentes biopsicosociodemográficos. Para la realización de este estudio se consideraron los requisitos éticos de E. Emanuel. Resultados: en promedio 3,8 (D.E = 0.66) los trabajadores/as de la UPC participante de este estudio, perciben sobrecarga mental al realizar su trabajo. Conclusión: la sobrecarga mental de trabajo, pudiera generar consecuencias en su salud física y mental de los enfermeros/as participantes, por lo que es prioritario tomar medidas tendientes a mantener una carga mental adecuada.

${ }^{*}$ Enfermera. Doctora en Enfermería. Académica Departamento de Enfermería Universidad Católica del Maule. Email: pceballos@ucm.cl
Palabras claves:

Carga Mental, Salud Laboral, Enfermería 


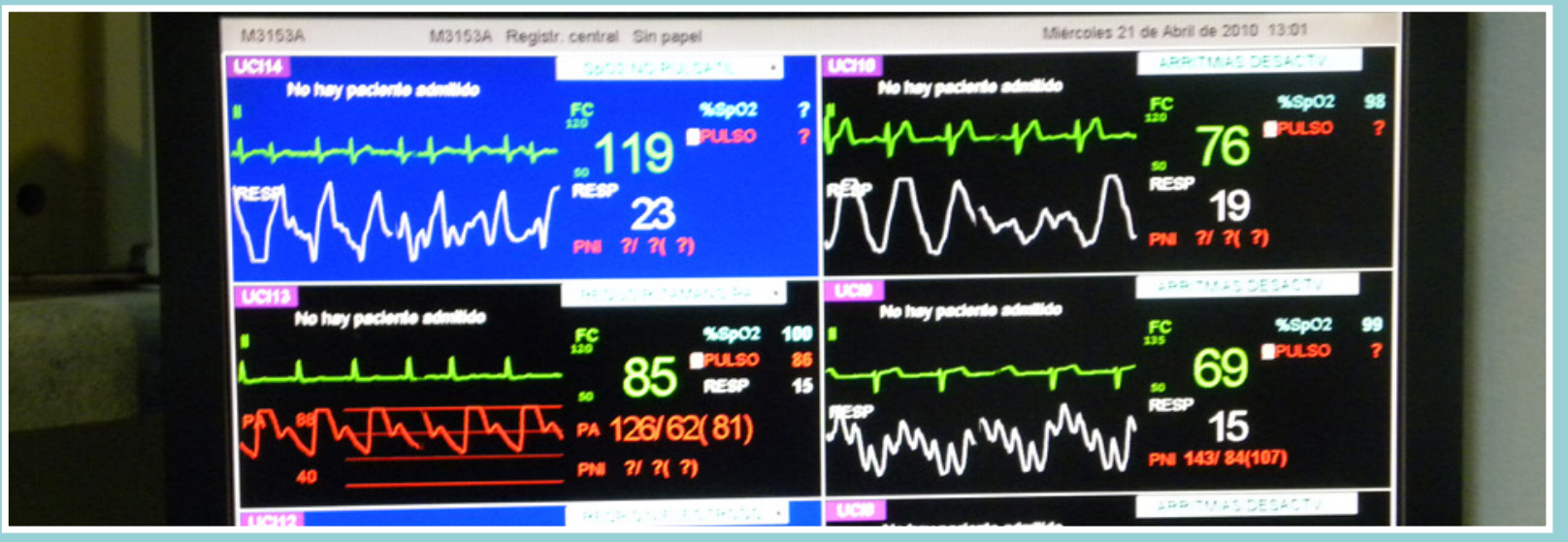

\section{INTRODUCCIÓN}

De forma general, el mundo laboral ha evolucionado de una elevada carga física, al aumento progresivo de la carga mental (1). Este cambio se visualiza en todos los sectores laborales, sin embargo, se agudiza en los trabajadores/as del sector servicios (e.j trasporte; educación, revendedores; Sanitarios, entre otros). Dentro de los trabajadores/as sanitarios, quienes se desempeñan en Unidades Críticas (Unidades de Cuidados Intensivos e Intermedios), pudieran estar más expuestos/as a estas modificaciones del entorno laboral por la complejidad del ambiente en que estos/as profesionales desarrollan su trabajo. Con el fin de demostrar empíricamente este fenómeno, se han revisado estudios desde el paradigma cualitativo y cuantitativo, realizadas en Chile y el extranjero. En los hallazgos bibliográficos, se observa percepción de falta de reconocimiento tanto de la jefatura como de los pares, bajo apoyo social y sobrecarga mental de trabajo, posicionando este último aspecto, el que más afecta a dichos profesionales. Según autores, dicho constructo reviste cada vez más importancia la seguridad y salud en el trabajo, a medida que la tecnología se ha ido introduciendo en la totalidad de los entornos, imponiendo mayores demandas cognitivas a los trabajadores. Además que presenta un impacto en la efectividad del desempeño humano ${ }^{(2-3)}$. Investigadores refieren que son los enfermeros/as son los más citados en estudios de agravios de la salud mental, especialmente por sobrecarga de trabajo (4). Es por ello que para el Consejo Internacional de Enfermería (CIE), es prioritario mantener ambientes de trabajo saludables que permitan la entrega de cuidados eficientes y eficaces ${ }^{(5)}$. Una de las estrategias para conseguir este objetivo es medir los niveles de carga mental en los diversos ámbitos de desempeño, con la finalidad de obtener resultados empíricos que visibilicen la problemática y con estos, los responsables de las empresas puedan modificar las funciones de los puestos de trabajo con el fin de reducir el nivel de carga mental de los trabajadores/as, y por esta vía mejorar la satisfacción y salud de sus funcionarios ${ }^{(6)}$. Expertos muestran que existen diferentes metodologías de medición de la Carga Mental de Trabajo CMT. Por nombrar algunas mediciones fisiológicas, objetivas y subjetivas. Se expone que las mediciones subjetivas son adecuadas, ya que miden la percepción del trabajador/a respecto al nivel de CMT generado por su desempeño. Al ser la CMT un constructo multidimensional dichas escalas o cuestionarios, responden a este criterio. Uno de ellos, Escala Subjetiva de Carga Mental de Trabajo (ESCAM), es el que se utilizó para este estudio piloto y será desarrollado en la descripción del instrumento ${ }^{(6-7)}$. Por lo anterior, el objetivo de esta investigación fue determinar la percepción de Carga Mental de Trabajo CMT en los enfermeros/as de la UPC de un Hospital Regional en Chile. 


\section{METODOLOGÍA}

Estudio de diseño descriptivo, trasversal y exploratorio. Se censó a diez enfermeras/os que se desempeñan en un turno de UPC de un Hospital Regional de Chile. Para la realización de este "estudio piloto", se utilizaron dos instrumentos: (a) la Escala Subjetiva de Carga Mental de Trabajo (ESCAM), desarrollada por el equipo integrado por las profesoras de la Universidad de La Laguna, Santa Cruz de Tenerife, España ${ }^{(6)}$. Para el uso de dicha escala se solicito autorización a los autores. ESCAM incluye 20 ítems, cada uno de ellos tiene 5 posibilidades de respuesta, en donde 1 corresponde a muy bajo y 5 muy alto. Dichos ítems se distribuyen en cinco dimensiones: Cantidad y complejidad de la información que el trabajador utiliza en su puesto de trabajo. Demandas cognitivas del trabajo realizado, referidas a atención, concentración, memorización, entre otras. Organización temporal del trabajo, comprende aspectos referidos a tiempo requerido, tiempo disponible, pausas y ritmo de trabajo. Características de la tarea, en referencia al número de tareas realizadas, complejidad de las mismas, interrupciones, demoras, recuperación de retrasos, consecuencia de errores. Consecuencias para la salud del trabajador, derivadas de un desequilibro en la carga mental de trabajo. ESCAM tiene una consistencia interna de 0,75 y fue validada para Chile en trabajadores sanitarios ${ }^{(18)}$.

(b) Antecedentes biopsico sociodemográficos, información referente a aspectos sociodemográficos (sexo, edad, estado civil, cualificación o formación), condición laboral (puesto, antigüedad, experiencia requerida, entre otros) y condiciones ambientales de trabajo (iluminación, ruido, temperatura).

La recolección de los datos se realizó en Diciembre del 2010. La organización, el procesamiento y análisis de los datos, se realizo con el software SPSS versión 15.0 para Windows $X P$, lo que permitió un análisis descriptivo de la información a través de frecuencias, medidas de tendencia central y de variabilidad (Media y D.E), para cada una de las dimensiones del instrumento utilizado. Para la realización de este estudio se consideraron los requisitos éticos de $E$. Emanuel ${ }^{(19)}$.

\section{RESULTADOS Y DISCUSIÓN}

El $88.9 \%$ de los participantes son mujeres y el $11.1 \%$ son hombres. Los resultados coinciden con otros estudios, en donde el porcentaje de mujeres se encuentra entre el $85 \%$ y un $77 \%$ respectivamente ${ }^{(12-13)}$. El promedio de edad de los participantes del estudio es de 34.8 años. Dicho resultado no es coincidente con la literatura, ya que el promedio de edad, en los dos estudios encontrados esta sobre los 40 años ${ }^{(12-13)}$, esto pudiera ser causa de la reciente contratación de profesionales de enfermería en la UPC estudiada, otra posible explicación al respecto, sería que se tomó solamente un turno para la aplicación de ESCAM, por lo que los profesionales de mayor edad podrían estar distribuidos en otras rotaciones. Por otra parte, el $77.8 \%$ de los enfermeros/as son solteros/as y el $44.4 \%$ de ellos tienen por lo menos un hijo/a, para estas dos variable no se encuentra en las referencias algún dato con el que se pueda contrastar los resultados obtenidos, pero se podría inferir que el gran porcentaje de solteros (as), es debido a la edad de los participantes, igual explicación para el número de hijos.

En relación a la situación laboral, se puede afirmar que el $66.7 \%$ de la muestra son enfermeras clínicas, las que se desempeñan en turno de 12 horas y el $33.3 \%$ son enfermeras(os) supervisores, los que trabajan en turnos de 9 horas. Presentan como promedio de antigüedad en el hospital 6.4 años y como promedio de antigüedad en el cargo 4.3 años, al compararlo con un estudio colombiano, no coincide con el tiempo de antigüedad en el cargo, ya que sobre el $70 \%$ de los participantes presentó 9 años o más de tiempo en el cargo ${ }^{(13)}$, en el estudio realizado es menor de 5 años promedio, la justificación a este aspecto, es la contratación de nuevos profesionales hace 2 años aproximadamente por ampliación de las Unidades intermedias de la UPC.

Con respecto a las condiciones ambientales de trabajo, $55.6 \%$ perciben que la temperatura de la Unidad en donde se desempeñan es inadecuada, este aspecto no coincide con una investigación brasileña, en donde el equipo de enfermería indica en un $8.9 \%$ alteraciones con la temperatura por inestabilidad ${ }^{(20)}$, este hecho se puede explicar por la diferencia geográfica en donde se llevaron a cabo los estudios, ya que brasil es una zona geográfica con altas temperaturas, que requiere en forma permanente aire acondicionado, adecuada ventilación, entre otros aspectos. En cuanto a la iluminación del puesto de trabajo, el $55.6 \%$ de los entrevistados indican que es intermedia, lo que coincide con dos investigaciones brasileñas, que indican alteraciones en la iluminación en un $38 \%$ y $15 \%$ respectivamente ${ }^{(10,20)}$, esto se fundamenta en el hecho en las UPC, la luz utilizada generalmente es artificial y pudiera ser considerada insuficiente por parte del equipo especialmente al momento de realizar procedimientos. El $66.6 \%$ de los profesionales refiere que 
el ruido de la unidad es inadecuado o muy inadecuado, la percepción de ruido en este grupo de profesionales es mayor que el obtenido en un estudio realizado el 2010, en donde los profesionales encuestados indican en un $30.8 \%$, la exposición a ruidos molestos ${ }^{(10)}$, esto se puede deber a que el estudio fue realizado en una unidad de menor complejidad, en donde la presencia de tecnología (Ventiladores mecánicos, bombas de infusión, monitores, entre otros), es menor que en las UPC. Al consultar sobre el espacio en su puesto de trabajo un $78.7 \%$ indica que es inadecuado o muy inadecuado, al igual que en estudios brasileños donde el $53.9 \%$ y $25 \%{ }^{(10,20)}$, indican un espacio físico inadecuado. Finalmente se les consulta por las condiciones higiénicas (Olores, polvos, contaminantes, entre otros), del puesto de trabajo, en donde un $55.5 \%$ indica que es inadecuado o muy inadecuado, este aspecto se evalúa en estudios extranjeros $(10,20)$, pero la percepción es mayor en esta investigacion. La explicación a esto pudiera ser el escaso espacio físico, por lo que los contaminantes se concentran en la unidad, la cantidad de químicos a utilizar, la preparación de medicamentos, etc.

En la Tabla $N^{\circ} 1$, la media de carga mental de trabajo permite señalar que los profesionales de enfermería encuestados perciben que las demandas cognitivas y la complejidad de la tarea desarrollada, les genera sobrecarga mental de trabajo. Esto significa que el trabajador percibe que debe realizar un gran esfuerzo a nivel cognitivo para desempeñar su puesto, exigencias elevadas de memorización, concentración, toma de decisiones, entre otras (6). Resultado similar al reportado en un estudio realizado en Chile, a personal de un centro de salud mental, en donde el $78.6 \%$ percibe una alta exigencia cognitiva en la realización del trabajo ${ }^{(21)}$. Así mismo, un estudio realizado al personal de enfermería de recuperación de anestesia reporta como resultado que el trabajo es minucioso y requiere de atención permanente cuando en la unidad hay usuarios" (22). Sin duda en las UPC, los profesionales de enfermería, están sometidos a la toma de decisiones permanentes y de forma inmediata, con un alto grado de memorización, entre otros lo que justifica la percepción de sobrecarga mental.

Tabla N 1: Puntuaciones de los/as enfermeros de UPC, en el Factor Demandas cognitivas y complejidad de la tarea.

\begin{tabular}{lcc}
\hline Factor Demandas cognitivas y complejidad de la tarea & Media $(x)$ & $D . E$ \\
\hline El nivel de esfuerzo o concentración mental que requiere mi trabajo & 4.44 & 0.72 \\
\hline $\begin{array}{l}\text { La cantidad de memorización de información y materia que se } \\
\text { requiere en mi trabajo es }\end{array}$ & 4.56 & 0.52 \\
\hline $\begin{array}{l}\text { El grado de complejidad de la información que debo utilizar en mi } \\
\text { trabajo es }\end{array}$ & 4.44 & 0.52 \\
\hline $\begin{array}{l}\text { El nivel de esfuerzo mental necesario para evitar los errores en mi } \\
\text { trabajo es }\end{array}$ & 4.00 & 1.58 \\
\hline $\begin{array}{l}\text { El nivel de ambigüedad de las decisiones a tomar en mi trabajo es } \\
\text { Habitualmente en mi puesto de trabajo el número de decisiones } \\
\text { que debo tomar es }\end{array}$ & 4.33 & 1.65 \\
\hline $\begin{array}{l}\text { Carga Mental de factor } \\
\text { nara }\end{array}$ & 4.22 & 0.52 \\
\hline
\end{tabular}

En la Tabla $N^{\circ} 2$, se observa que en la mayoría de los ítems, la media de la carga mental de trabajo, reporta la percepción de sobrecarga Mental de Trabajo. Solo un ítem es reportado con una percepción de carga media, dicho resultado se podría sustentar en la "creencia" que los profesionales de enfermería que se desempeñan en estas unidades, poseen un alto grado de preparación, especialización continua, etc, por lo que la incorporación de nuevas tecnologías no debiera alterar su percepción de Carga Mental. Las características de la tarea de los profesionales de UPC participantes, entendida

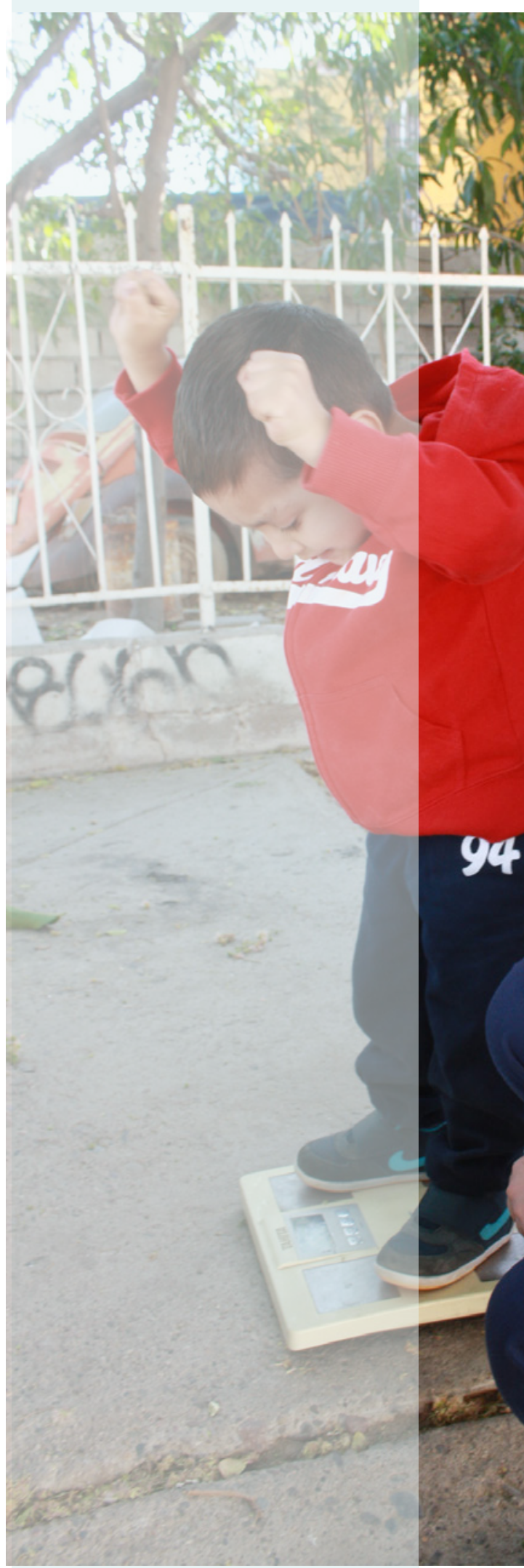


De esta manera, que el trabajador/a percibe que tiene mediana autonomía para modificar su ritmo de trabajo ${ }^{(24)}$, lo que es positivo y actuaría como un amortiguador para los otros factores de la CMT.

Tabla $N^{\circ}$ 4: Puntuaciones de los/as enfermeros de UPC, en el Factor Ritmo de trabajo

\begin{tabular}{lcc}
\hline Factor Organización temporal & Media $(x)$ & D.E \\
\hline $\begin{array}{l}\text { Es posible variar mi ritmo de trabajo sin perturbar el trabajo de mi } \\
\text { sección }\end{array}$ & 2.22 & 1.09 \\
\hline $\begin{array}{l}\text { Además de las pausas reglamentarias en trabajo me permite } \\
\text { hacer algunas pausas cuando lo necesito }\end{array}$ & 3.00 & 1.32 \\
\hline $\begin{array}{l}\text { En mi trabajo, puedo cometer algún error sin que incida en forma } \\
\text { crítica sobre los resultados del trabajo }\end{array}$ & 4.44 & 1.01 \\
\hline Carga mental del factor & 3.22 & 1.12
\end{tabular}

Se observa en la Tabla $N^{\circ}$, que este factor es generador de sobrecarga mental, es decir, el enfermero/a de UPC siente mucho cansancio y le cuesta relajarse después de terminar su jornada laboral (24). Al contrastar los resultados obtenidos con otras investigaciones, se visualiza que, el percibir sobrecarga mental, trae consecuencias para la salud del profesional de enfermería de UPC. Un estudio cualitativo realizado en Brasil, muestra que el profesional de enfermería refiere un agotamiento intenso ${ }^{(17)}$. Similares resultados muestra un estudio, el cual suma problemas como anemia, leucopenia, inmunidad disminuida, intolerancia, desánimo y estrés, por parte de los enfermeros/ as, participantes ${ }^{(26)}$. Lo expuesto hace visible que la sobrecarga mental, no solo afecta la producción de la institución, el riesgo de sufrir accidentes o la calidad del cuidado entregado a los usuarios, sino que también, la salud de los profesionales de enfermería. Este aspecto es relevante en relación a siguientes evaluaciones de CMT, el fin de mejorar el bienestar y la satisfacción de dichos profesionales, para una entrega de cuidados de calidad.

Tabla $N^{\circ}$ 5: Puntuaciones de los/as enfermeros de UPC, en el Factor Consecuencias para la salud

\begin{tabular}{lcc|}
\hline Factor Organización temporal & Media $(x)$ & $D . E$ \\
\hline Al final de la jornada de trabajo me siento agotado & 4.67 & 0.70 \\
\hline $\begin{array}{l}\text { Me siento agotado cuando me levanto por la mañana y tengo } \\
\text { que enfrentarme a otro día de trabajo }\end{array}$ & 3.33 & 1.87 \\
\hline El cansancio que me produce mi trabajo es & 4.33 & 0.70 \\
\hline Tengo dificultades para relajarme después del trabajo & 3.56 & 1.13 \\
\hline Carga mental del factor & 3.97 & 0.63
\end{tabular}

En la Tabla $N^{\circ} 6$, se observa que el segundo factor es percibido con una mayor sobrecarga mental por parte de los profesionales encuestados. En promedio, los trabajadores/as de la UPC participantes de este estudio perciben sobrecarga mental al realizar su trabajo. Esto se relaciona con otros estudios realizados en Chile, en donde el $78 \%$ de los encuestados percibe sobrecarga mental (21). En relación a estudios en unidades de alta complejidad, indican que los mayores niveles de Carga mental de trabajo se observan en las unidades pre-hospitalarias, seguido de la UCI pediátrica y UCI de adultos ${ }^{(13)}$. Estas referencias demuestran la complejidad relacionada al acto del cuidar de otros, del trabajo en equipo y de liderar la gestión del cuidado entre otros aspectos que debe llevar a cabo el profesional de enfermería que laboran en las Unidades de Paciente Crítico, tanto a nivel nacional como internacional. 
Tabla $N^{\circ}$ 6: Puntuaciones de los/as enfermeros de UPC, en cada uno de los factores de Carga Mental de Trabajo

\begin{tabular}{lcc}
\hline Carga de Mental total & Media $(x)$ & D.E \\
\hline Demandas cognitivas & 4.22 & 0.48 \\
\hline Características de la tarea & 4.39 & 0.60 \\
\hline Organización temporal & 2.96 & 0.12 \\
\hline Ritmo de trabajo & 3.22 & 1.12 \\
\hline Consecuencias para la salud & 3.97 & 0.63 \\
\hline Carga Mental de Trabajo Total & 3.8 &
\end{tabular}

\section{CONCLUSIONES}

- El objetivo de este estudio fue determinar la percepción de la Carga Mental de Trabajo de los enfermeros/as de una Unidad de Pacientes Críticos. Posterior a la aplicación de ESCAM, se concluye que el grupo entrevistado percibe Sobrecarga Mental al realizar sus actividades, lo que pudiera generar consecuencias en su salud física y mental, por lo que es prioritario tomar medidas tendientes a mantener una carga mental adecuada. Específicamente, el factor Característica de la tarea, es el reporta una mayor sobrecarga en este grupo de profesionales, lo que sirve de guía para la implementación o proposición de medidas de mejora del entorno laboral en UPC.

- Los estudios localizados referentes a la materia son escasos, lo que genera la necesidad de indagar e investigar sobre el tema, sumado a la divulgación de sus resultados, con el fin de conocer la realidad con respecto a la Carga Mental de Trabajo de los profesionales de enfermería de las Unidades de Paciente Crítico u otros lugares de desempeño.

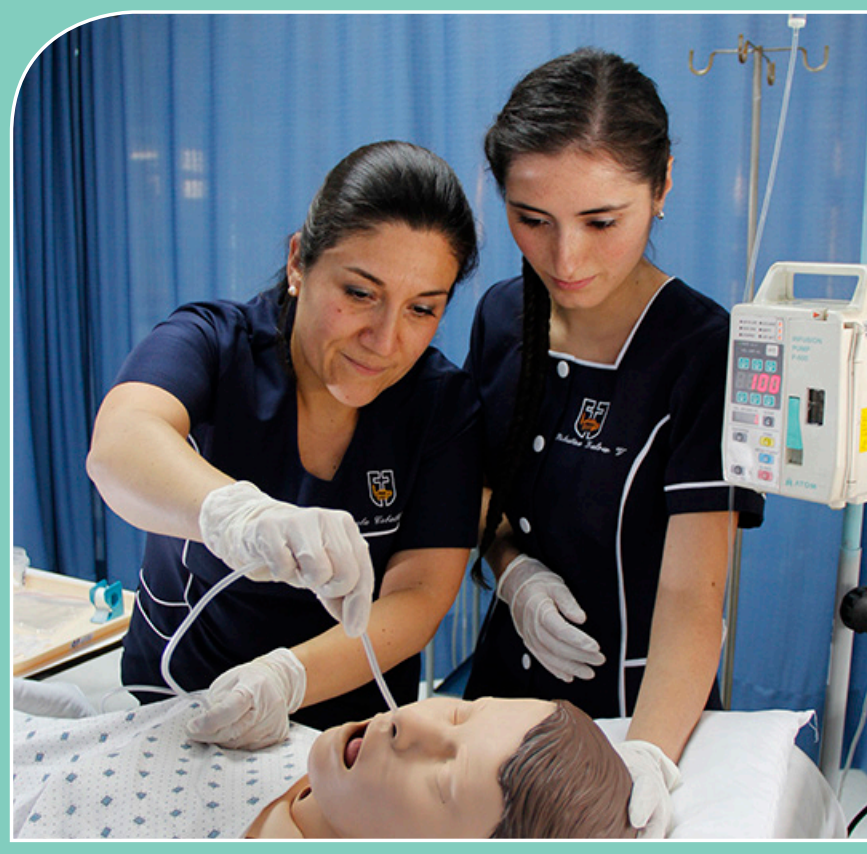

\section{REFERENCIAS}

1. Gil-Monte P. Riesgos psicosociales en el trabajo y salud ocupacional. Rev Perú Med Exp Salud Pública. 2012; 29(2): 237-41.

2. Ferrer R, Dalmau I. Revisión del concepto de carga mental: evaluación, consecuencia y proceso de normalización. Anuario de psicología. 2004; 35(4): 521-45.

3. Díaz C. Actividad laboral y carga mental de trabajo. Ciencia y trabajo. 2010; 12(36); 281-92.

4. Robazzi M, Mauro M, Dalri R, Da Silva $L$, Secco I, Pedrao L. Exceso de trabajo y agravios mentales a los trabajadores de la salud. 2010; 26(1): 52-64).

5. Consejo Internacional de Enfermería (CIE).
Las enfermeras una fuerza para el cambio. Un recurso vital en salud. Ginebra, Suiza: International Council of Nurses; 2014.

6. Rolo G, Díaz D, Hernández E. Desarrollo de una escala subjetiva de carga mental de trabajo (ESCAM). Revista de psicología del trabajo y de las organizaciones. 2009; 25(1): 29-37.

7. Díaz Ramiro E, Rubio ValdehitaS, Martín García J, Luceño Moreno L. Estudio psicométrico del índice de Carga Mental NASA-TLX con una muestra de trabajadores españoles. Revista de psicología del trabajo y de las organizaciones. 2010; 26(3):191 - 99.

8. Apud E, Gutiérrez M, Maureira F, Lagos $S$, Meyer F, Chiang M. Guía para la evaluación de trabajos pesados. $1^{\circ}$ ed. Trama impresores. 2002.

9. Mauro M, Muzi C, Guimaraes R, Mauro C. Riesgos ocupacionales en salud. Rev Enferm UERJ. 2004; 12: 338-45.

10. Mauro $M$, Da Paz A, Mauro $C$, Pihneiro M, Silva V. Condicoes de trabalho da Enfermagem nas enfermeiras de um hospital Universitario. Esc Anna Nery Rev Enferm. 2010; 14(2):244-52.

11. Castro M, Farias S. A producto científica sobre riescos ocupacionais a que estao expostos os trabalhadores de enfermagem. Esc Anna Nery Rev Enferm. 2008; 12(2): 364-69. 


\section{REFERENCIAS}

12. Briceño C, Herrera R, Enders J, Fernández A. Estudios de riesgos ergonómicos y satisfacción laboral en el personal de enfermería. Revista de salud pública. 2005; 9(1): 53-9.

13. González Gutiérrez J, Moreno Jiménez B, Garrosa Hernández E. Carga Mental y fatiga en servicio especiales de enfermería. Revista latinoamericana de psicología. 2005; 37(3): 477 - 92.

14. Hacker W. Carga mental de trabajo. En: Enciclopedia de salud y seguridad en el trabajo, Organización Internacional del trabajo (OIT); 2001. [Citado 20 ago 2015]. Disponible en: http://www. insht.es/InshtWeb/Contenidos/Documentacion/TextosOnline/ EnciclopediaOIT/tomo1/29.pdf

15. Cañas J. Personas y maquinas. El diseño de su interacción desde la ergonomía cognitiva. Ed. Pirámide. Madrid, España; 2004.

16. Young M, Stanton N. Mental Workload: Theory. Measurement and Application. En: W. Karwowski. International Encyclopedia of Ergonomics and Human Factors. Londres; Taylor\& Francis; 2001.

17. Shumizu H, Couto D, Harmann E. Placer y sufrimiento en trabajadores de enfermería en una UTI. Rev Latino-am Enferm. 2011; 19(3).

18. Ceballos V, Paravic T, Burgos M, Barriga O. Validación de Escala Subjetiva de Carga Mental de Trabajo en funcionarios/as universitarios. Cienc Enferm. 2014; 20(2):72-84.).

19. Emanuel E, Wendler D, Grady C. What make clinical research ethical?. JAMA. 2000; 283(20): 2701-11.

20. Benatti M, Nishide V. Elaboracao e implemetacao do mapa de riscos ambientais para prevencao de accidentes do trabalho em uma unidade de terapia intensiva de um hospital universitario. Rev Latino-am Enfermagem. 2000; 8(5):13-20

21. Aguirre Martínez R. Valuación ergonómica de carga mental y factores de riesgo psicosocial del trabajo, en profesionales, técnicos y administrativos de un centro de rehabilitación de salud mental privado. Revista Chilena de Terapia Ocupacional. 2010; 10: 85 - 97.

22. Bozzo E, Chávez E, Ozonas M, Tomasina F. Condiciones de trabajo de enfermería del departamento recuperación post anestésica hospital de clínica Dr. Manuel Quintela. Universidad de la República. Revista Uruguaya de enfermería. 2006; 1(1): 5 - 13.

23. Díaz D, Hernández E, Rolo G. Carga mental de trabajo. España: Ed. Síntesis; 2012.

24. Díaz D, Hernández E, Rolo G, Galván E, Fraile, M, Loayssa G. Escala Subjetiva de carga mental (ESCAM). Tenerife: Instituto Canario de Seguridad Laboral. [On line]. 2010. [Acceso 20 ago 2015]. Disponible en: http://www.gobiernodecanarias.org/cicnt/icasel/documentos/ manualcarga_mental.pdf.

25. Guimares R, Mauro M, Méndez R, De Melo A, Da Costa T. Factores ergonómicos de risco e de trabalho: um estudo caso-controle. Rev Bras Epidemiol. 2006; 8(3): 282 - 94.

26. Maia J, Barbosa E, Da Costa A. Estresee, factor di risco para a saúde do enfermeiro em centro de terapia intensiva. R Enferm UERJ. 2006; 14(4): $580-85$.

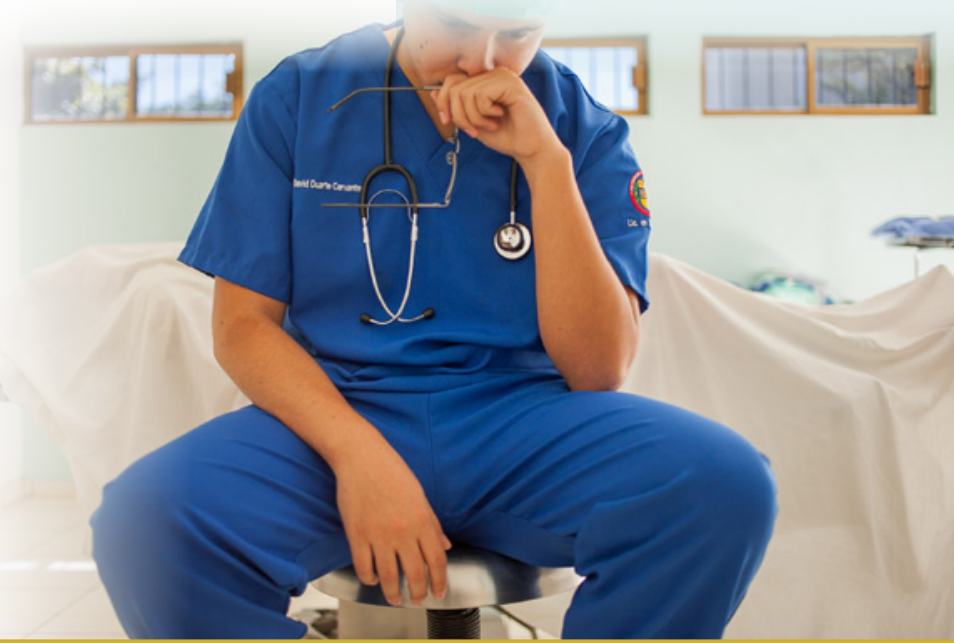

